\title{
A METHODOLOGY TO REDUCE VARIABILITY DURING VACUUM INFUSION WITH OPTIMIZED DESIGN OF DISTRIBUTION MEDIA
}

\author{
Hatice S. Sas, Pavel Šimáček, Suresh G. Advani* \\ Department of Mechanical Engineering, \\ Center for Composite Materials, \\ University of Delaware, DE 19716
}

\begin{abstract}
Seemann Composites Resin Infusion Molding Process (SCRIMP) is a widely used version of Vacuum Assisted Resin Transfer Molding (VARTM) in which a highly permeable layer (distribution media) is placed on top of the dry preform to distribute the resin with very low flow resistance to reduce the filling and hence the manufacturing time. The flow patterns during filling may vary from part to part due to the variability associated with the material, part geometry, and layup of the assembly, which may result in race-tracking channels. The process is considered as reliable and robust only if the resin completely saturates the preform despite changing filling patterns caused by flow disturbances.
\end{abstract}

The resin flow pattern can be manipulated with a tailored distribution media layout as it does impact the flow patterns significantly. The continuous distribution media layout over the entire part surface works well for very simple geometries with no to little potential for race-tracking along the edges. In this study we address complex cases, which require placement of an insert within the assembly, which will introduce race-tracking along its edges, and hence uniform placement of distribution media over the entire top surface will fail to yield a void free part. We introduce a methodology using a predictive tool to design an optimal shape of distribution media, which accounts for the flow variability introduced due to race-tracking along the edges of the inserts. This iterative approach quickly converges to provide the placement of distribution media on selective areas of the preform surface that ensures complete filling of the preform despite the variability. This approach has been validated with an 
experimental example and will help mitigate risk involved in manufacturing complex composites components with Liquid Molding.

\section{INTRODUCTION}

The term Liquid Composite Molding (LCM) is used for closed mold processes in which a dry fibrous preform is placed in the mold cavity and the mold is closed [1]. Then, a liquid resin is impregnated through the preform to fill the empty spaces between the fibers in the mold cavity. Resin Transfer Molding (RTM) is one of the most commonly used process in which the resin is impregnated within the empty pores of the preform in a two-sided closed mold. The Vacuum Assisted Resin Transfer Molding (VARTM) is the other commonly used LCM process because of its ability to manufacture large scale, low cost composites. In VARTM, the preform is placed on a one-sided tooling surface and the other side is sealed with a vacuum bag. Vacuum is applied at the vent line to remove the air from the mold cavity, which induces fiber compaction under atmospheric pressure and draws resin into the mold cavity through the injection line. After resin arrives at the vent, the injection is discontinued but the vacuum is maintained until the resin cures allowing one to de-mold the final composite part [2]. To speed up the process, a highly permeable material called as the distribution media (DM) is placed in between the top layer of the fabric and the vacuum bag in the process SCRIMP. The use of DM decreases the fill time because the resin first flows through the DM layer and then impregnates through the thickness direction $[3,4]$. Thus, SCRIMP eliminates the disadvantage of long fill times for VARTM. However, SCRIMP might lead to formation of voids (especially at the leading edge of the flow front) and for complex geometries and parts containing impermeable inserts [5]. 
To ensure successful impregnation of the resin to cover all the empty spaces within the dry preform and manufacture composite parts without macro-scale voids (dry spots), process models and simulations have been developed to predict the process parameters (inlet/vent locations) [6-8]. These process models are based on resin flow through porous media governed by Darcy's Law $[9,10]$ which relates the pressure gradient to average resin velocity within the preform

$$
\overline{\mathrm{u}}=-\frac{\mathrm{K}}{\underline{\mu}} \cdot \nabla \mathrm{P}
$$

where $\overline{\mathrm{u}} \mathrm{u}$ is the volume averaged velocity, $\mathrm{K}$ is the permeability tensor that describes the fiber preform resistance to the flow of impregnating resin in various directions, $\mu$ is the viscosity and $\nabla \mathrm{P}$ is the pressure gradient experienced by the resin. The permeability of the preform can be numerically predicted, or experimentally measured.

A variety of effects can cause random local variations in fibrous preform porosity or architecture. However this will not change the filling pattern very dramatically [11]. On the other hand, flow disturbances introduced due to race-tracking as a result of open channels created between the mold and preform edges or along sharp bends in reinforcement or between preform and inserts in the mold can change the filling pattern significantly. This potentially could allow the resin to reach the vent line before impregnating the entire preform which will result in a large macro-void (dry spot) within the part resulting in the part being scraped or re-worked. [12-17]. In RTM, since the mold is a two-sided closed cavity resin racing along the mold edges is more common than VARTM in which one-sided mold is sealed with vacuum bag. In VARTM, race-tracking will be more prominent along the boundaries of the inserts in the mold or around sharp bends. The process design should be able to address the possible effect of the flow disturbances because of race-tracking to manufacture composite structures without macro-scale voids (dry spots). One could manage the flow 
disturbances because of the race-tracking by placing flow runner channels in strategic locations intentionally [18]. Other approach is to introduce active control algorithms with sensors in the mold that can correct for the flow once race-tracking is detected to ensure successful filling of the preform [20-23]. Also, studies have been performed to identify the disturbance locations and develop scenario-based solutions [24-27] using active and passive flow control. However, actively controlled scenario based solutions require expensive tooling, placement of robust sensors at the strategic locations and complicated hardware for flow control that is integrated with the injection equipment. Also these tools can be readily implemented with RTM as one can direct the resin flow by introducing more injection gates and locally changing the pressure field. This is more difficult to implement in VARTM $[28,29]$. Hsiao et al. proposed use of distribution media, which is more appropriate for VARTM processing, as a flow control tool and the race-tracking possibility is handled by placing flow channels [19].This study also proposes a passive control approach by designing a DM layout for that particular case. The tailored distribution media for the part geometry should ensure successful filling despite the presence of race-tracking that might change from one injection to the next for manufacturing the same part. At the same time, it must guarantee acceptable infusion time in all feasible cases. This is presented in this work.

\section{METHODOLOGY AND IMPLEMENTATION}

First, given the part geometry along with insert locations, one must identify all possible race-tracking locations within the part and create possible scenarios, which take into account all possible permutations of race-tracking that may occur during the impregnation process. To determine a single optimal layout of DM for all these possible scenarios, a discrete optimization method is adopted. Discrete definition is generated by dividing the surface of the preform (where one places the DM) into a finite number of regions. Optimal solution finds the 
regions where one should place the DM such that for all scenarios the resin will arrive at the vent last after having impregnated the entire preform. Mathematically, this is done by prescribing the cost function that will minimize the region with no resin in the mold. Evaluation of the cost function is performed with an existing numerical simulation called Liquid Injection Molding Simulation (LIMS) [30] which simulates the flow in any complex geometry in Liquid Molding. LIMS output provides the empty region after each fill based on the inputs of preform and DM permeability, race-tracking strengths along the edges and predefined inlet and vent locations. Race-tracking strength, which is defined as the ratio of the permeability of the race-tracking channel to the permeability of the preform in the channel direction, is modeled by generating one-dimensional mesh using LIMS which interfaces with the part geometry mesh. Thus, the permeability of the one-dimensional mesh elements representing the race-tracking channels are set to the value of race-tracking strength multiplied by permeability of the preform along the race-tracking channel direction.

\subsection{Tree Search Algorithms}

Gradient descent method is a first-order algorithm to find a local minimum of a function. The method starts with an initial guess of the solution and the gradient of the function at that point is evaluated. The solution is stepped in the negative direction of the gradient and the process is repeated until the algorithm converges to a zero gradient. This method works for the objective functions for which the gradient can be evaluated. If the variables used in the objective function are only a finite or discrete set of values, discrete optimization should be applied. The discrete optimization problem can be defined as a set, $S$ of finite possibilities that satisfies the objective function. The objective function of that provides local minimum, $\mathrm{x}_{\mathrm{opt}}$ for all elements of the set $S$, 
$\mathrm{f}\left(\mathrm{x}_{\text {opt }}\right) \leq \mathrm{f}(\mathrm{x})$ for all $\mathrm{x} \in \mathrm{S}$

Discrete optimization can be used for different problems such as such as VLSI layouts, robot motion planning, test pattern generation, and facility location [31].

The search of the optimal solution with discrete optimization consists of computationally expensive problems. As Koft [32] states there are two parameters that indicates the complexity of the searches: the branching factor of the problem space and the depth of solution of the problem. The branching factor represents the number of the new states that are generated and analyzed at each depth. The depth of the search is the distance between the initial state and goal state. There are two basic tree search algorithms; breadth-first search (BFS) and depth-first search (DFS). In Figure 1.a, a sample tree layout is given and there are two goal nodes satisfying the objective function, $\mathrm{H}$ and $\mathrm{T}$ which are the two local minimum satisfying the user defined tolerance. BFS expands all the states one step away from the initial state until a goal state is reached and converges to node $\mathrm{H}$, before node T (Figure 1.b). DFS explores a path all the way to a leaf before backtracking and exploring another path. Therefore, only path of nodes from the initial node to the current node must be stored in order to execute the algorithm. DFS will find the node T, before the node H (Figure 1.c) [33].

As the BFS converges, the solution at the minimum depth is found (Figure 1.b). When a DFS succeeds, the solution may not be at the minimum depth (Figure 1.c). For a large tree, BFS may have large memory requirements because BFS stores all possible nodes at each depth. Thus, BFS convergences may take long time to reach the solution, goal node [33]. For the solution for DM layout design the minimum depth is not a concern but the memory is. So, DFS is adopted by generating regions on the preform which represents the finite 
solution set and the cost function is the unfilled area. Different race-tracking scenarios yield different unfilled regions and our goal is a DM layout design that results in a successful filling without voids for all possible race-tracking scenarios. Thus, the approach adopted is to first investigate all possible RT scenarios and implement the DFS algorithm to find a DM layout solution for the worst filling RT scenario defined by largest unfilled region. Then, this updated DM layout solution is used for the remaining cases of the RT scenarios and new worst filling RT scenario is identified. If this new worst filling satisfies the acceptable tolerance, the algorithm stops. If not, the DM layout is updated (superimposing on the DM layout from the previous iteration). This procedure is repeated until the layout satisfied the filling of the mold within an acceptable tolerance for all permutations of RT cases with the DFS algorithm.

The objective function is to minimize the unfilled region for all possible scenarios. Fill time can also be included into the objective function. In the current approach this is solved by testing for allowable time limit. That is included in the objective function as a Heaviside function - as long as the time to fill is below the gel time of the resin it receives no consideration. If the fill time is higher than the gel time, the solution cost function instantly becomes infinite and is not considered. This methodology is explained in more details in the next sub-section by an example problem.

\subsection{Pedagogical Example}

In Figure 2, a pedagogical example is presented to explain the methodology. For prescribed inlet and vent locations, three different race-tracking options are identified, the permutations of which could result in $2^{3}=8$ different race-tracking scenarios (Figure 2.a). In the first step, all possible eight scenarios are simulated without the use of DM using the simulation engine LIMS and the worst case of filling is identified by the maximum percentage of 
unfilled region (dry spots or voids) by halting the simulation when the resin arrives at the selected vent. The simulation output is the fill factor for each node. Initially all nodes have zero fill factor and as the resin reaches a node the fill factor is updated to 1 . The success of filling can either be measured by calculating the porous volume via integration of the fill factor values over the preform domain, or using the number of empty nodes (output of LIMS simulation). Both approaches are tested and the results were within a few percent of the values provided by empty node approximation. Therefore the number of empty nodes are used as it is the most efficient method in terms of computational performance.

For this example "Case 8" resulted in largest unfilled region (14.1\%- worst case) as shown in Figure 2.a. The methodology suggests finding the DM layout for the worst case "Case 8" first. DM layout solution is found by generating the discrete domain, such as dividing the domain into six areas. Then, six separate simulations are conducted with DM placed in each of the six regions on top of the preform successively and the percentage of unfilled region is recorded for each placement as shown in Figure 2.b. As seen from Figure 2.b, by placing the $\mathrm{DM}$ on the right bottom corner provides the best filling option $(0.1 \%$ void $)$ out of the six configurations and that DM layout results in a mold filling that is within the user defined tolerance prescribed which is usually around 1 to $2 \%$ as allowing for bleeding may reduce that region to less than $1 \%$ and in some cases if the void is close to the vent, it will get flushed out with the resin. Next, this DM layout that provides successful fill for "Case 8" is applied to the remaining seven scenarios and it is found that the "Case 1" which has no race-tracking along any edge has one of the largest unfilled region $(11.5 \%)$ in this case as shown in Figure 2.c and is the worst case out of the six. 
In order to find a successful filling solution for "Case 1", the DM design from the previous solution is retained and the approach to conduct six simulations with an additional DM patch placed in each of the six areas successively is repeated and the resulting unfilled area percentages are recorded. It should be noted that the $5^{\text {th }}$ configuration represents placement of 2 layers of DM on the right bottom corner. The best filling solution for the "Case 1" results in $7.3 \%$ voids, which is greater than the tolerance (Figure 2.d). Therefore, the successive placement of DM in the six regions is repeated for "case 1" while retaining both the DM patches placed in the earlier trials (Figure 2.e). This trial results in zero voids and successful filling for the case in which the DM is placed in the middle of the top half while maintain the two DM layers from the previous trials as shown in Figure 2.e. Finally, the updated design, which resulted in no voids for "Case1", is used to perform mold filling simulation for the remaining seven cases and the results are shown in Figure 2.f, which shows that the worst case scenario is "Case 8 " with void region of $0.5 \%$ which is within the tolerance limit. Thus, this represents the final DM layout design which will provide successful resin impregnations for all 8 scenarios possibly expected during manufacturing with the specified locations for the gate and vent. For this example and for this gate and vent location we were successful in finding the DM layout, which worked for all 8 cases. However, if this was not the case, one would continue with this algorithm of adding a region and testing the cases until all six regions were covered with DM. After covering all the regions if still one could not find a solution, then the number of regions is increased from six to eight (or ten or twelve) and the algorithm is repeated. If the gate and vent location are changed, one would expect the DM design to change as well. 


\subsection{Adapted Algorithm}

In the previous section methodology the solution is sought by using six regions. However, one may not converge to a solution with the given regions. Thus, the adapted algorithm is developed not only to find an optimized DM layout for all possible RT scenarios for a given region but also to be able to update the number of the regions, if necessary. In Figure 3, the flowchart of the algorithm is presented. First, the domain is divided into $2 \mathrm{n}$ regions and solution is sought as explained in the pedagogical example. At the end of the Discrete Optimization (DO) routine the filling of the best case is selected and if that filling still has voids more than the tolerance limit; the number of DM region is increased by 1 and DO is repeated until the voids are within the tolerance limit. If the number of DM regions (m) is equal to the regions of the domain (2n) and still the voids are more than the tolerance limit, then the number of regions in increased by a factor of 2 and the entire cycle is repeated as shown in Figure 3.

\subsection{Partition Method}

The presented methodology optimizes DM layout on discrete domain. The discrete domain is generated by dividing the domain, the top surface of the preform where the DM is to be placed, into finite number of regions. This is accomplished by Matlab ${ }^{\circledR}$ built in k-means++ algorithm [34]. The k-means clustering is a partitioning method. The coordinates of the domain forms the data set and clustering divides the data into $\mathrm{k}$ clusters and indexes the cluster. The objective is to have the points in the same cluster being as close as possible to each other and as far as possible from points in other clusters. It is an iterative method that minimizes the sum of the distances from each object to its cluster centroid. Figure 4 presents an example region for the given domain.

\section{EXPERIMENTAL VALIDATION}

After the optimum DM layout is obtained via the proposed algorithm, one can experimentally test the design. In order to visualize the movement of the 
flow front through the fibrous medium with time and observe the filling of the preform, the test set up as shown in Figure 5, was used. After the preform along with a steel insert is placed on the acrylic table, it is sealed with a vacuum bag and resin at atmospheric pressure is introduced from one end through a line gate while drawing a vacuum at the other end. The resin propagates within the preform due to the pressure gradient of one atmosphere and the flow front movement is captured via the flow visualization camera system along with the time stamp.

In this experiment due to its Newtonian characteristic, corn syrup with dark cloth dye and water is used as the simulated resin to create a clear contrast between the dark resin front and the white glass fibrous porous media. The experiments are carried out using 8 layers of $50 \mathrm{~cm} \times 50 \mathrm{~cm}$ Plain Weave E-glass and distribution media made of polypropylene. The impermeable metal $1 \mathrm{~mm}$ thick square metal insert $(20 \mathrm{~cm} \times 20 \mathrm{~cm})$ is placed in the center of the $4^{\text {th }}$ layer (Figure 6.a). After adding the remaining 4 layers of fabric, the DM layer is placed on the top and the preform is sealed and vacuum is applied (Figure 6.b). Using the set-up, the flow front positions along the top and the bottom are recorded with time stamps. Possible race-tracking can occur along the insert edges as the preform may not completely close the gap around the insert. The material properties of the corn syrup mixture, fiber preform and the DM were measured and are listed in Table 1 [35]. Since, it has been shown that replacing the compliant material and variable permeability by the "effective" value results in the same flow patterns and, if the effective value is properly established, very good time correlation is obtained [36]. 


\section{RESULTS AND DISCUSSION}

\subsection{Experimental Validation}

The methodology presented in section 2 was applied to the mold geometry presented in Figure 6 to find the DM layout to be placed on top of the preform so no voids are created [30]. The mesh representing the preform domain is shown in Figure 7.a with the line inlet at the left side and vent on the right side with 4 race-tracking possibilities $\left(2^{4}=16\right.$ scenarios $)$. Then the algorithm presented in Figure 3 is executed for placement of DM. The algorithm started to seek solution with 2 regions, 4 regions and 6 regions (Figure 4), respectively, and the algorithm converged to solution with 8 regions as shown in Figure 7.b. The algorithms finds the optimal DM layout design given in Figure 7.c as a Cshape DM layer to be placed on the left side of the preform that provides successful filling for all 16 different possible scenarios. For the optimum layout of this case the algorithm executed 4 test LIMS simulations of 16 scenarios and 5 iterative LIMS simulations were run for the placement of DM on 8 defined regions. For the LIMS simulations the boundary conditions are; no flow through the boundary, zero pressure at the flow front and prescribed pressure at the node/s in the inlet region. The algorithm managed to solve that optimization problem with 104 total LIMS simulations in 18 minutes on a PC computer with the user defined tolerance of $2 \%$ voids (unfilled volume).

Manufacturing using the conventional way by covering nearly the entire preform (95\%) with DM (leaving 5\% gap at the end so the resin does not straight end up in the vent through the DM) results in voids within the part [37]. Figure 8 shows the resin flow front patterns along the top and the bottom of the part in 4 of the possible 16 scenarios that can occur due to permutations of race-tracking effects along the edges of the insert. These results are contrasted with the designed DM design which do not result in any voids for all 16 scenarios of which only the 4 ones are displayed. Use of DM on $95 \%$ of the top 
layer decreases fill time but as it can be seen from the flow front profiles at the bottom (Figure 8.a) that large voids do form for this DM layout. Simulations with the optimized DM design clearly show that successful filling without entrapping any voids despite different flow front profiles (Figure 8.b). Use of tailored DM design also saves DM material as one does not need to cover the entire top of the mold with DM.

The design is also tested experimentally to validate it. In Figure 9.(a) the formation of the dry spot underneath the metal insert at the bottom using the 95\% DM is presented. Figure 9.(b) and Figure 9.(c) shows the experimental and one of the scenario of numerical flow front progression at the top and bottom of the preform at time intervals of 20 seconds, respectively. As seen in the experimental comparison, formation of the dry spot is prevented. The experimental fill time is 221 seconds. The numerical fill times are in the range of 183 seconds to 273 seconds. The experimental flow front profiles shows straight flow front lines as the corn syrup reaches the vent and the filling is complete without voids. However, the profiles do not exactly match with any of the 16 numerical scenarios, though they are "close". This can be explained by several factors. First and most importantly, the assigned of the "race-tracking strength" value for the simulations is not exactly known value but is an estimate. The strength of the race-tracking is the ratio of permeability along the racetracking line to preform permeability in the direction of the race-tracking line. For the simulations this value is kept at a very high value, 1000, so the DM design will work for smaller race-tracking strength values as well. Second, there might be additional deviations between numerical model and the experiment because of the experimental errors during material characterization $[38,39]$. However, the flow pattern is reasonably captured despite these discrepancies and the proposed solution is able to fill the mold for all scenarios even if the times do not exactly match. 


\subsection{Complex Geometries}

The proposed methodology is tested with a complex geometry with corners and edges. As seen in Figure 10, an optimized DM 1 design is presented for a trailer of a truck. For this geometry there are 10 different RT channel possible whose permutations will yield 1024 different scenarios. The algorithm is used with defined inlet and vent locations and DM layout solution is sought that considers all 1024 scenarios and converges to the DM design that provides successful filling for all 1024 cases. For this geometry the convergence is tested with 2, 4, 6, 8, 10, 12 and 14 regions, respectively, and the algorithm convergences to the design given in Figure 10 with 14 regions.

If the entire top surface is covered with the DM, in 886 scenarios out of 1024 the unfilled volume will be more than $1 \%$ with the maximum unfilled volume being $20 \%$. If the optimized DM design is used, in all 1024 cases the void fraction is less than $1 \%$, the largest being $0.79 \%$. Figure 11 shows void regions for the three representative examples out of 1024 for the case of entire top surface being covered by the DM on the left hand side and with the optimized DM design on the right hand side that is free of voids. Figure 12 presents the time contours comparison for fully covered DM situation and the optimized DM design for the same three scenarios. The fill time in the optimized DM case is slightly higher but it is robust enough to provide successful filling despite the variability introduced from possible race-tracking. The lowest and the largest fill time for full DM case was 3710 and 3458 seconds where as for the designed DM case, the fill time was 10459 and 8413 respectively from all 1024 scenarios. Additionally, Figure 13 gives the pressure distribution data at the time when the resin reaches the vent location for full DM and DM layout design. Besides the improvement in the filling the DM design also provides uniform pressure along the preform that yields uniform volume fraction and uniform material properties. 
In order to examine the effect of mesh size on the DM design and CPU time four mesh sizes starting with 0.05 m element size and halving it 3 times was investigated. As given in Figure 14, except for the first coarse mesh, the rest of the meshes used converged to the same DM design but the CPU time increased exponentially with finer mesh size with tolerance of $5 \%$ unfilled area. For all those 4 cases, the algorithm converged when the domain is divided into 2 regions and $34(16+2+16)$ LIMS simulations are performed for each case. However, for the very coarse mesh (mesh size $0.05 \mathrm{~m}$ ) required tolerance is not satisfied with the $\mathrm{C}$ shape DM design as for the other cases and convergence to the DM design is achieved when the domain is divided into 14 regions which make the CPU time needed for the very coarse mesh the largest. Hence it is important to ensure that the mesh is fine enough to obtain DM design convergence with fewer numbers of divisions.

\section{CONCLUSION}

In this study a methodology is introduced to design an optimum distribution media layout that makes the process robust by successful filling for all possible disturbances caused by different race-tracking scenarios around inserts. Depth First Search, a tree search, algorithm is adopted via discretization of the domain into finite regions to arrive at the DM design. The algorithm is demonstrated with an example and an experimental validation is presented.

\section{ACKNOWLEDGEMENTS}

This work is funded by National Science Foundation with grant number 0856399. Authors would like to thank Prof. Rakesh for this advice on the development of the methodology based on discrete optimization. 


\section{REFERENCES}

[1] Advani SG, Sozer M. Process Modeling in Composites Manufacturing. New York: Marcel Dekker, Inc.; 2003.

[2] Acheson JA, Simacek P, Advani SG. The implications of fiber compaction and saturation on fully coupled VARTM simulation. Compos Part A Appl Sci Manuf 2004;35:159-69. doi:10.1016/j.compositesa.2003.02.001.

[3] Han K, Jiang S, Zhang C, Wang B. Flow modeling and simulation of SCRIMP for composites manufacturing. Compos Part A Appl Sci Manuf 2000;31:79-86. doi:10.1016/S1359-835X(99)00053-6.

[4] Park CH, Woo L. Modeling void formation and unsaturated flow in liquid composite molding processes: a survey and review. J Reinf Plast Compos 2011;30:957-77. doi:10.1177/0731684411411338.

[5] Lawrence JM, Frey P, Obaid AA, Yarlagadda S, Advani SG. Simulation and Validation of Resin Flow During Manufacturing of Composite Panels Containing Embedded Impermeable Inserts With the VARTM Process. Polym Compos 2007;28:442-50. doi:10.1002/pc.

[6] Lee LJ, Young WB, Lin RJ. Mold filling and cure modeling of RTM and SRIM processes. Compos Struct 1994;27:109-20.

[7] Lim ST, Lee W Il. An analysis of the three-dimensional resin-transfer mold filling process. Compos Sci Technol 2000;60:961-75.

[8] Trochu F, Ruiz E, Achim V, Soukane S. Advanced numerical simulation of liquid composite molding for process analysis and optimization. Compos Part A Appl Sci Manuf 2006;37:890-902. doi:10.1016/j.compositesa.2005.06.003.

[9] Bear J. Dynamics of Fluids in Porous Media. New York: Elsevier; 1972.

[10] Dullien FAL. Porous media fluid transport and pore structure. San Diego, CA: Academic Press; 1992.

[11] Li J, Zhang C, Liang R, Wang B, Walsh S. Modeling and analysis of thickness gradient and variations in vacuum-assisted resin transfer molding process. Polym Compos 2008;29:473-82.

[12] Young W. Analysis of the edge effect in resin transfer molding. Compos Part A Appl Sci Manuf 1997;28A:817-22.

[13] Hammami A, Gauvin R, Trochu F, Touret O, Ferland P. Analysis of the Edge Effect on Flow Patterns in Liquid Composites Molding. Appl Compos Mater 1998;5:161-73.

[14] Lawrence JM, Fried P, Advani SG. Automated manufacturing environment to address bulk permeability variations and race tracking in resin transfer molding by redirecting flow with auxiliary gates. Compos 
Part A Appl Sci Manuf 2005;36:1128-41.

doi:10.1016/j.compositesa.2005.01.024.

[15] Bickerton S, Advani SG. Characterization and modeling of race-tracking in liquid composite molding processes. Compos Sci Technol

1999;59:2215-29.

[16] Lawrence JM, Barr J, Karmakar R, Advani SG. Characterization of preform permeability in the presence of race tracking. Compos Part A Appl Sci Manuf 2004;35:1393-405.

doi:10.1016/j.compositesa.2004.05.002.

[17] Devillard M, Hsiao K, Gokce A, Advani SG. On-line Characterization of Bulk Permeability and Race-Tracking During the Filling Stage in Resin Transfer Molding. J Compos Mater 2003;37:1525-41. doi:10.1177/002199803034459.

[18] Mohan R, Bickerton S, Shires D, Advani SG, Tamma K. Analysis and characterization of flow channel effects during the manufacturing of composites by resin transfer molding. Proc. Annu. Meet. Soc. Plast. Eng. ANTEC, Toronto: n.d.

[19] Hsiao K-T, Devillard M, Advani SG. Simulation based flow distribution network optimization for vacuum assisted resin transfer moulding process. Model Simul Mater Sci Eng 2004;12:S175-90. doi:10.1088/0965-0393/12/3/S08.

[20] Hsiao K, Gillespie JW, Advani G, Fink BK. Role of Vacuum Pressure and Port Locations on Flow Front Control for Liquid Composite Molding Processes 2001;22:660-7.

[21] Johnson RJ, Pitchumani R. Enhancement of flow in VARTM using localized induction heating. Compos Sci Technol 2003;63:2201-15. doi:10.1016/S0266-3538(03)00179-9.

[22] Lawrence JM, Hsiao K-T, Don RC, Simacek P, Estrada G, Sozer EM, et al. An approach to couple mold design and on-line control to manufacture complex composite parts by resin transfer molding. Compos Part A Appl Sci Manuf 2002;33:981-90. doi:10.1016/S1359835X(02)00043-X.

[23] Modi D, Correia N, Johnson M, Long A, Rudd C, Robitaille F. Active control of the vacuum infusion process. Compos Part A Appl Sci Manuf 2007;38:1271-87. doi:10.1016/j.compositesa.2006.11.012.

[24] Hsiao K-T, Advani SG. Flow sensing and control strategies to address race-tracking disturbances in resin transfer molding. Part I: design and algorithm development. Compos Part A Appl Sci Manuf 2004;35:114959. doi:10.1016/j.compositesa.2004.03.010.

[25] Devillard M, Hsiao K-T, Advani SG. Flow sensing and control strategies to address race-tracking disturbances in resin transfer 
molding - part II: automation and validation. Compos Part A Appl Sci Manuf 2005;36:1581-9. doi:10.1016/j.compositesa.2004.04.009.

[26] Nielsen D, Pitchumani R. Intelligent model-based control of preform permeation in liquid composite molding processes, with online optimization. Compos Part A Appl Sci Manuf 2001;32:1789-803. doi:10.1016/S1359-835X(01)00013-6.

[27] Lawrence J, Advani S. Dependence Map-Based Flow Control to Reduce Void Content in Liquid Composite Molding. Mater Manuf Process 2005;20:933-60. doi:10.1081/AMP-200060419.

[28] Alms J, Advani SG. Simulation and experimental validation of flow flooding chamber method of resin delivery in liquid composite molding. Compos Part A Appl Sci Manuf 2007;38:2131-41. doi:10.1016/j.compositesa.2007.06.011.

[29] Alms JB, Advani SG, Glancey JL. Liquid Composite Molding control methodologies using Vacuum Induced Preform Relaxation. Compos Part A Appl Sci Manuf 2011;42:57-65. doi:10.1016/j.compositesa.2010.10.002.

[30] Simacek P, Advani SG. Desirable features in mold filling simulations for Liquid Composite Molding processes. Polym Compos 2004;25:35567. doi:10.1002/pc.20029.

[31] Grama A, Kumar V, Member S. State of the Art in Parallel Search Techniques for Discrete Optimization Problems. IEEE Trans Knowl Data Eng 1999;11:28-35.

[32] Korf R. Depth-first iterative-deepening An optimal admissible tree search. Artif Intell 1985;27:97-109. doi:10.1016/0004-3702(85)900840.

[33] Grama A, Karypis G, Kumar V, Gupta A. Introduction to Parallel Computing. Harlow, England; New York: 2003.

[34] Arthur D, Arthur D, Vassilvitskii S, Vassilvitskii S. k-means++: The advantages of careful seeding. Proc Eighteenth Annu ACM-SIAM Symp Discret Algorithms 2007:1027-35. doi:10.1145/1283383.1283494.

[35] Lugo J, Simacek P, Advani SG. Analytic method to estimate multiple equivalent permeability components from a single rectilinear experiment in liquid composite molding processes. Compos Part A Appl Sci Manuf 2014;67:157-70. doi:10.1016/j.compositesa.2014.08.031.

[36] Simacek P, Advani SG. "Equivalent" Permeability and Flow in Compliant Porous Media. Submitted to Compos Part A Appl Sci Manuf.

[37] Heider D, Simacek P, Dominauskas A, Deffor H, Advani S, Gillespie JW. Infusion design methodology for thick-section, low-permeability preforms using inter-laminar flow media. Compos Part A Appl Sci Manuf 2007;38:525-34. doi:10.1016/j.compositesa.2006.02.016. 
[38] Bruschke M V, Advani SG. A Finite Element/Control Volume Approach to Mold Filling in Anisotropic Porous Media. Polym Compos 1990;11:398-405.

[39] Diallo ML, Gauvin R, Trochu F. Experimental Analysis and simulation of flow through multilayer fiber reinforcement in LCM. Polym Compos 1998;19:246-56. 


\section{Tables}

Table 1. Properties of E-glass fabric, DM and corn syrup

\begin{tabular}{ll}
\hline Parameter: & $\begin{array}{l}\text { Numerical } \\
\text { value: }\end{array}$ \\
\hline \hline Density of E-glass: & $2500\left[\mathrm{~kg} / \mathrm{m}^{3}\right]$ \\
Fiber volume fraction of E-glass: & $50 \%$ \\
Permeability of E-glass: & $\mathrm{K}_{\mathrm{xx}}=8.32 \mathrm{e}-11\left[\mathrm{~m}^{2}\right]$ \\
& $\mathrm{K}_{\mathrm{yy}}=5.88 \mathrm{e}-11\left[\mathrm{~m}^{2}\right]$ \\
& $\mathrm{K}_{\mathrm{zz}}=3.49 \mathrm{e}-12\left[\mathrm{~m}^{2}\right]$ \\
Permeability of race-tracking channel & $\mathrm{K}_{\mathrm{RT}} \mathrm{xx}=1000 \mathrm{xK} \mathrm{K}_{\mathrm{xx}}\left[\mathrm{m}^{2}\right]$ \\
& $\mathrm{K}_{\mathrm{RT}} \mathrm{yy}=1000 \mathrm{x} \mathrm{K}_{\mathrm{yy}}\left[\mathrm{m}^{2}\right]$ \\
Density of DM: & $946\left[\mathrm{~kg} / \mathrm{m}^{3}\right]$ \\
Fiber volume fraction of DM: & $15 \%$ \\
Permeability of DM: & $3.46 \mathrm{e}-09\left[\mathrm{~m}^{2}\right]$ \\
Viscosity of corn syrup & $100[\mathrm{cP}]$ \\
\hline
\end{tabular}




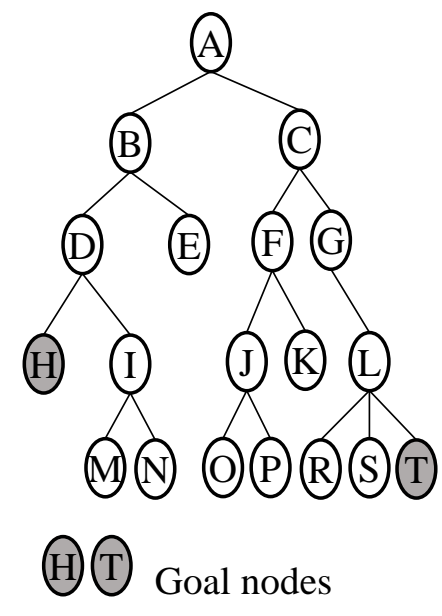

(a)

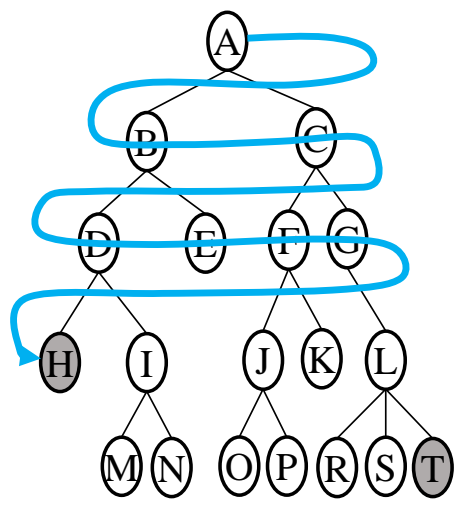

(b)

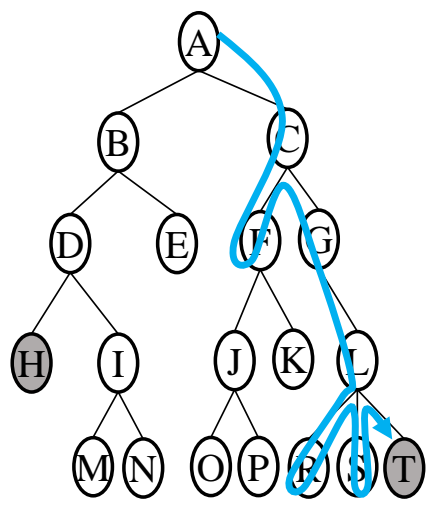

(c)

Figure 1. Tree search algorithms (a) example problem with two acceptable, $\mathrm{H}$ and $\mathrm{T}$, nodes, (b) Breadth-first search: finds node H, (c) Depth-first search: finds node T 


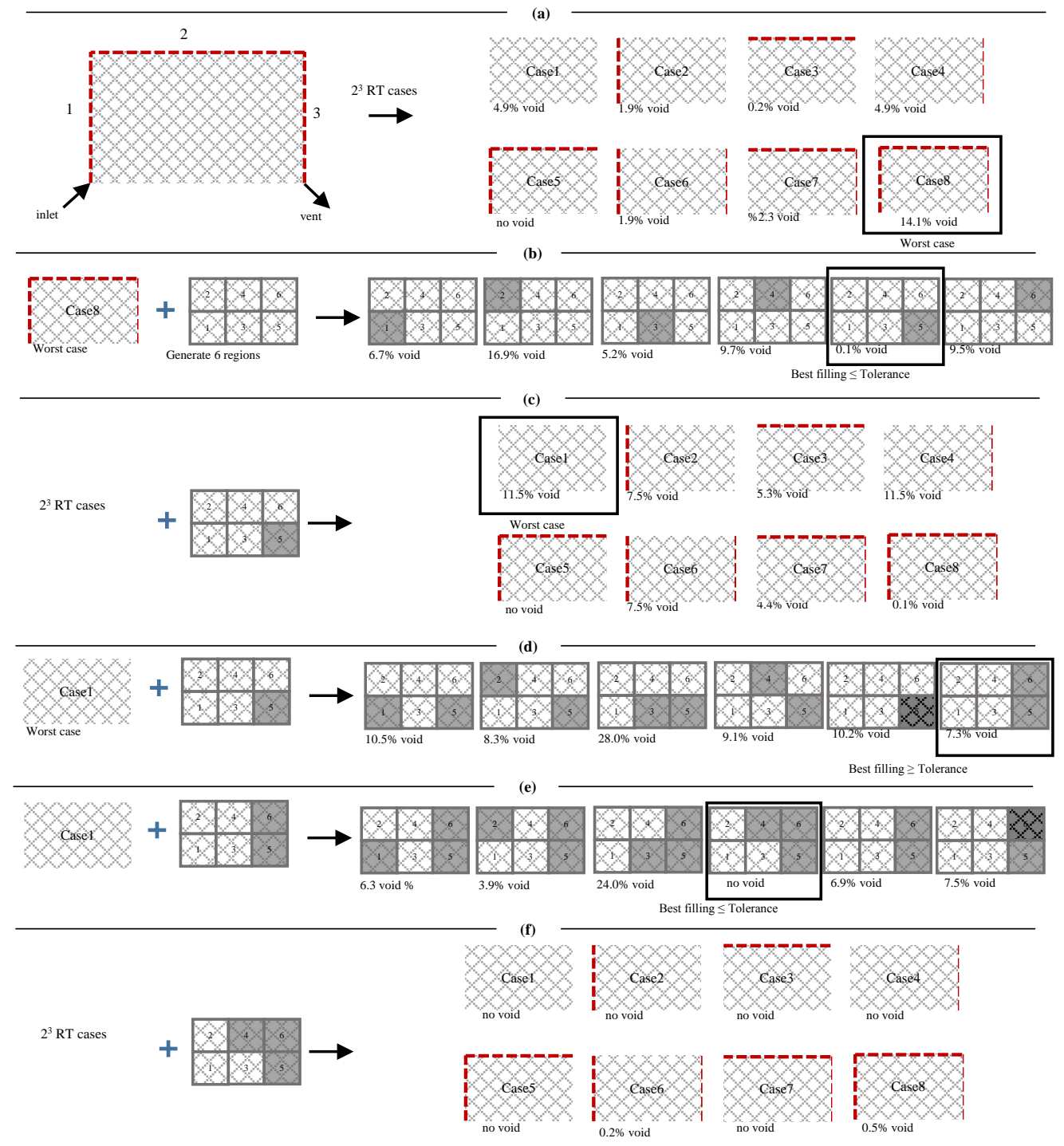

Figure 2. Example to explain the methodology to determine the optimal DM design using the DFS discretization method 


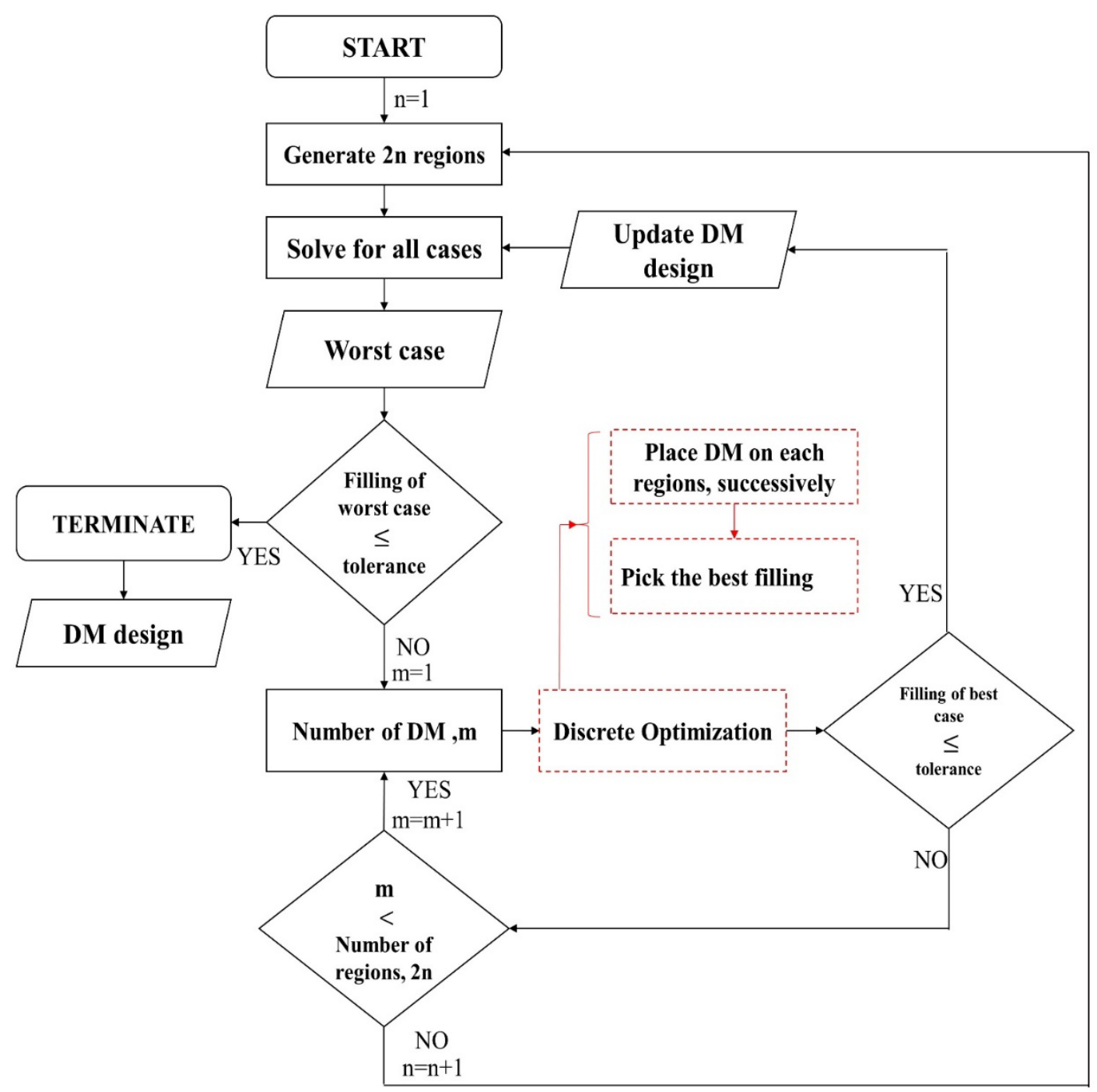

Figure 3. Flow chart of the algorithm to obtain optimal DM 


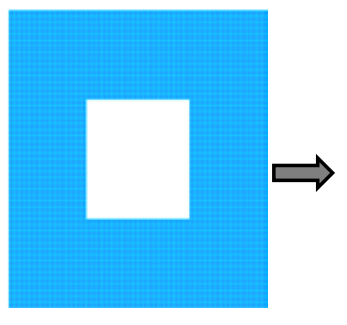

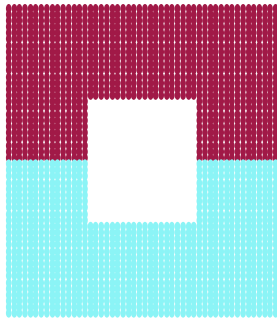

2 regions

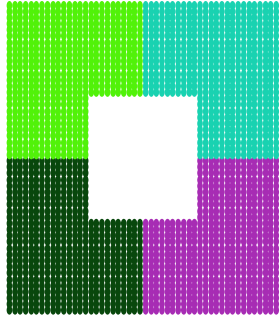

4 regions

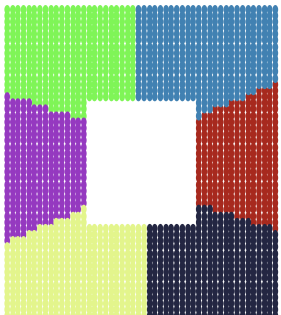

6 regions

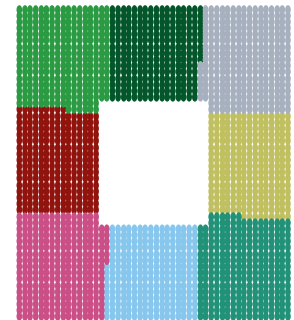

8 regions

Figure 4. Division of the domain with the built in k-means script in Matlab

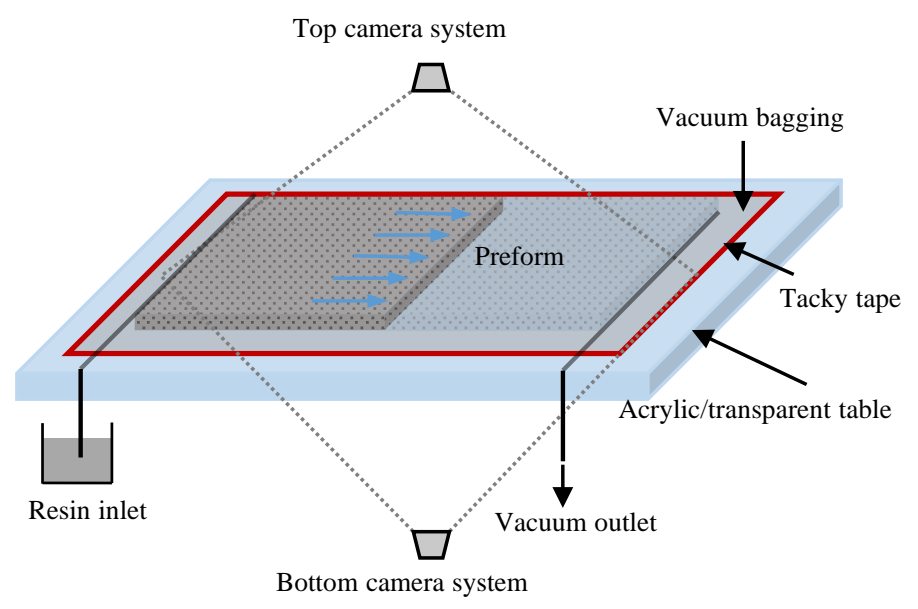

Figure 5. VARTM test set-up with two cameras system to record the flow front along the top and the bottom surface. 


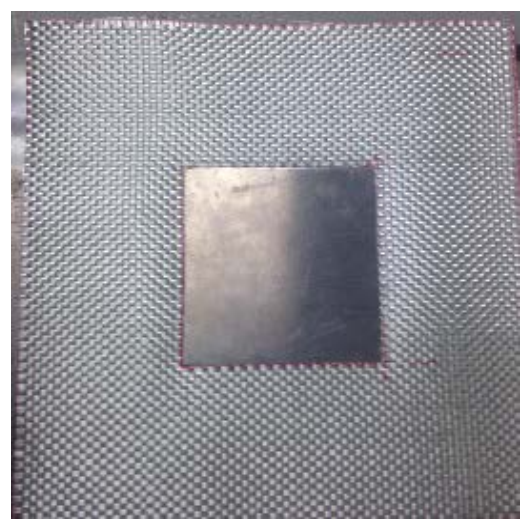

(a)

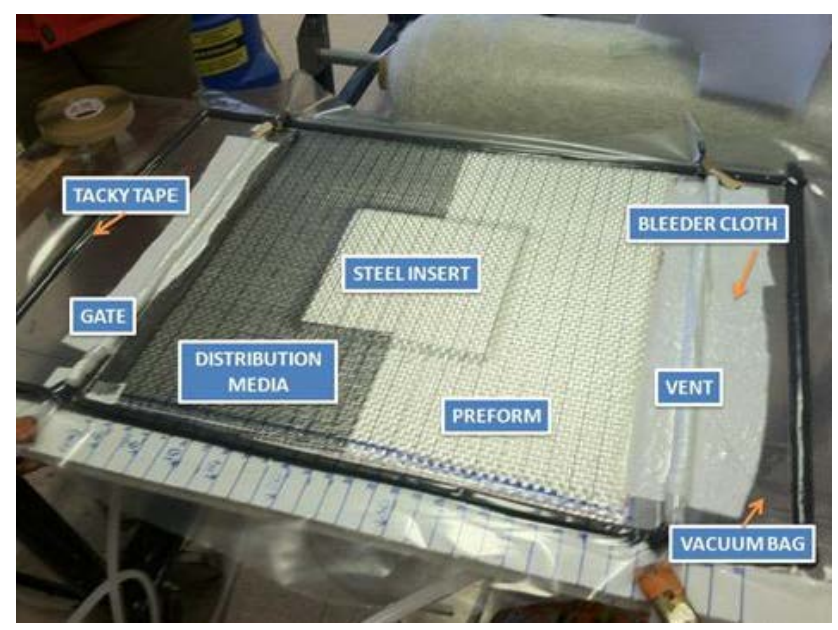

(b)

Figure 6. (a) 4th layer of the E-glass with metal insert placed in the center of the fabric, (b) Experiment layup under vacuum

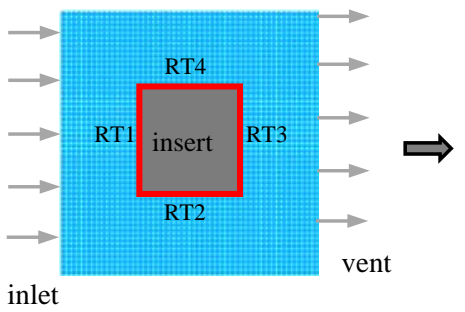

(a)

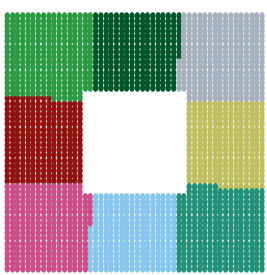

8 regions

(b)

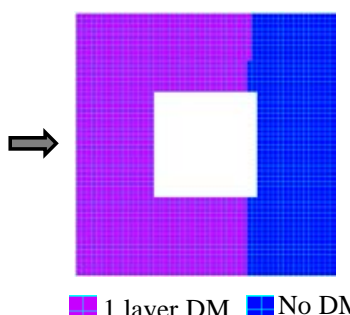

(c)

Figure 7. DM layout design (a) geometry with inlet/vent locations with 4 race-tracking possibilities along the insert edges creating 24=16 different scenarios (b) 8 regions for placement of distribution media when using discrete optimization, and (c) optimum DM design which resulted in successful filling for all 16 scenarios. 
(a) With 95\% DM
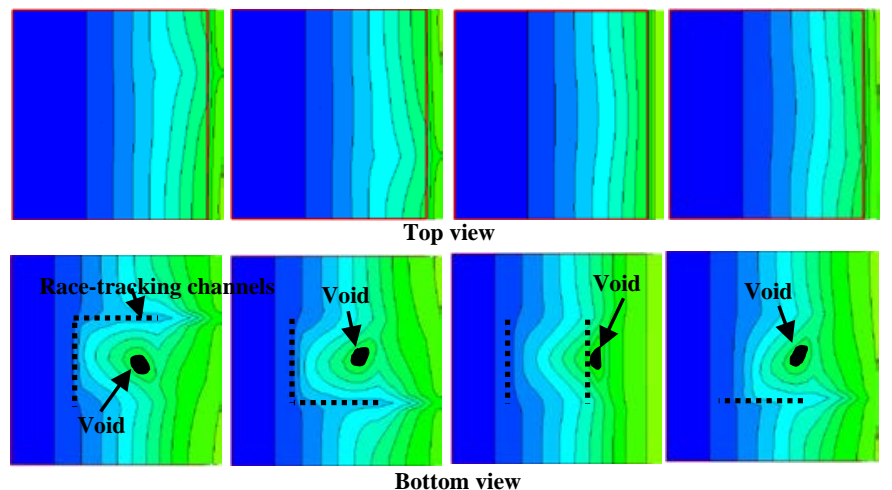

(b) With optimized Distribution Media
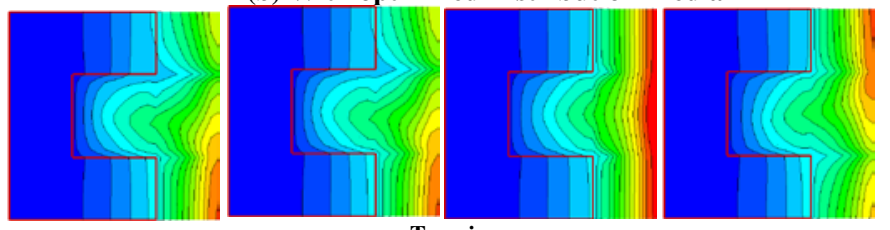

Top view
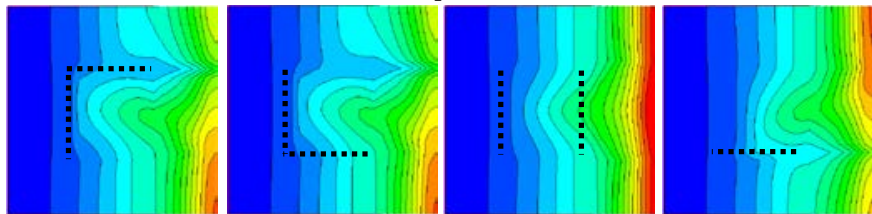

Bottom view

Time: 102030405060708090100110120130140150160

Figure 8. Numerical Solution of flow front profiles of the top and bottom views for 4 different race-tracking scenarios with time steps 10 seconds apart, (a) with 95\% of the top layer covered with DM, (b) with optimized DM design 
Top views

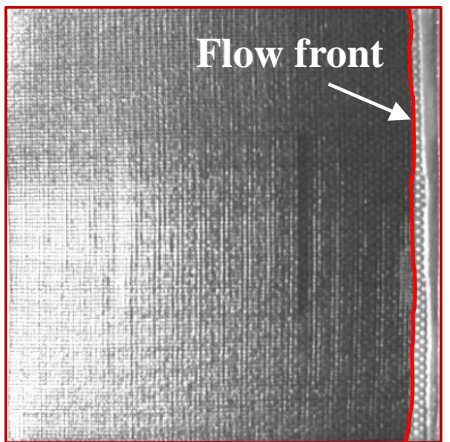

(a) With 95\% DM

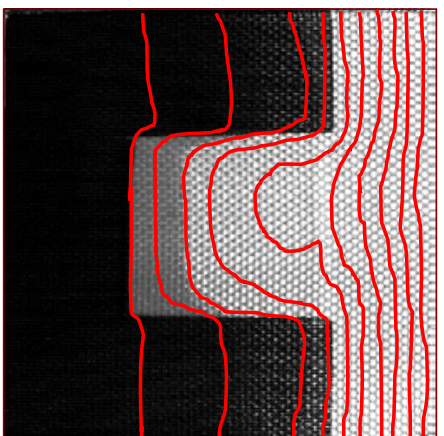

(b) With optimized DM
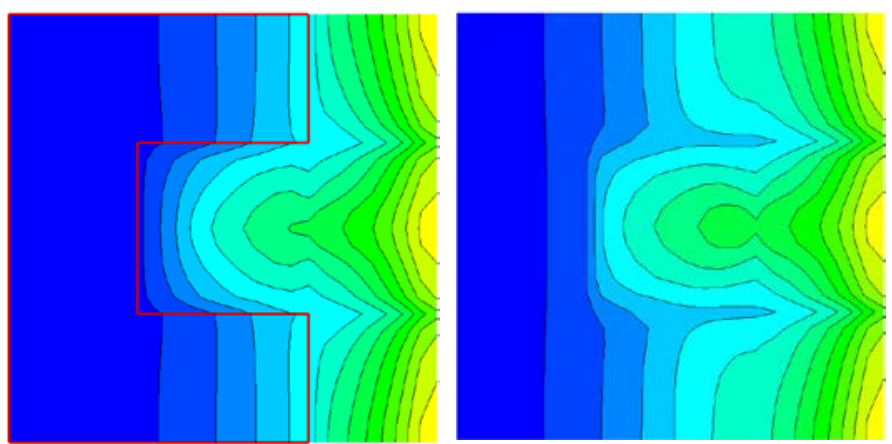

(c) Numerical with optimized DM

Figure 9. Top and bottom flow front profiles: (a) with 95\% $\mathrm{DM}$ at the time corn syrup reached the vent (experimental), (b) with optimized DM in 20 seconds apart with the background image of the experiment at 60 seconds (experimental), (c) one of the scenario of numerical simulation in 20 seconds apart 

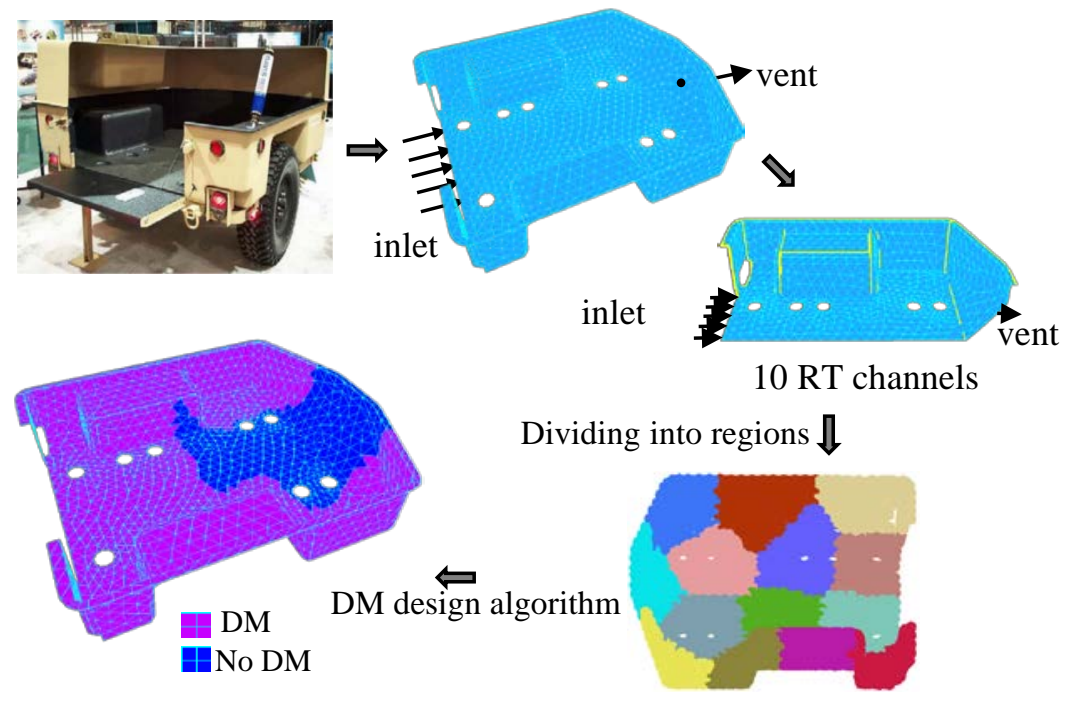

Figure 10. Optimized DM design of trailer geometry with 1024 different possible flow patterns 


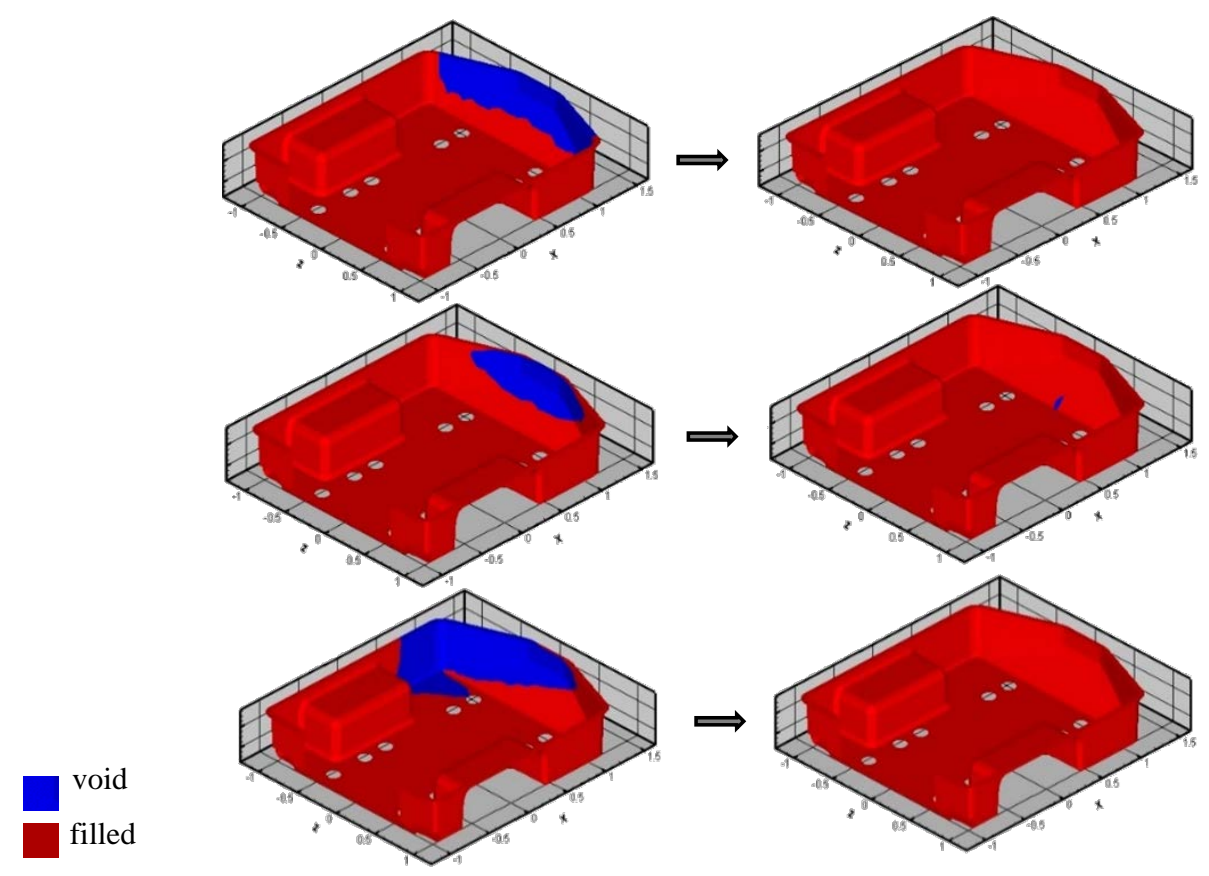

Figure 11. Void regions with full DM on top surface on the left hand side with optimized DM design on the right hand side for three representative scenarios from 1024 possible scenarios

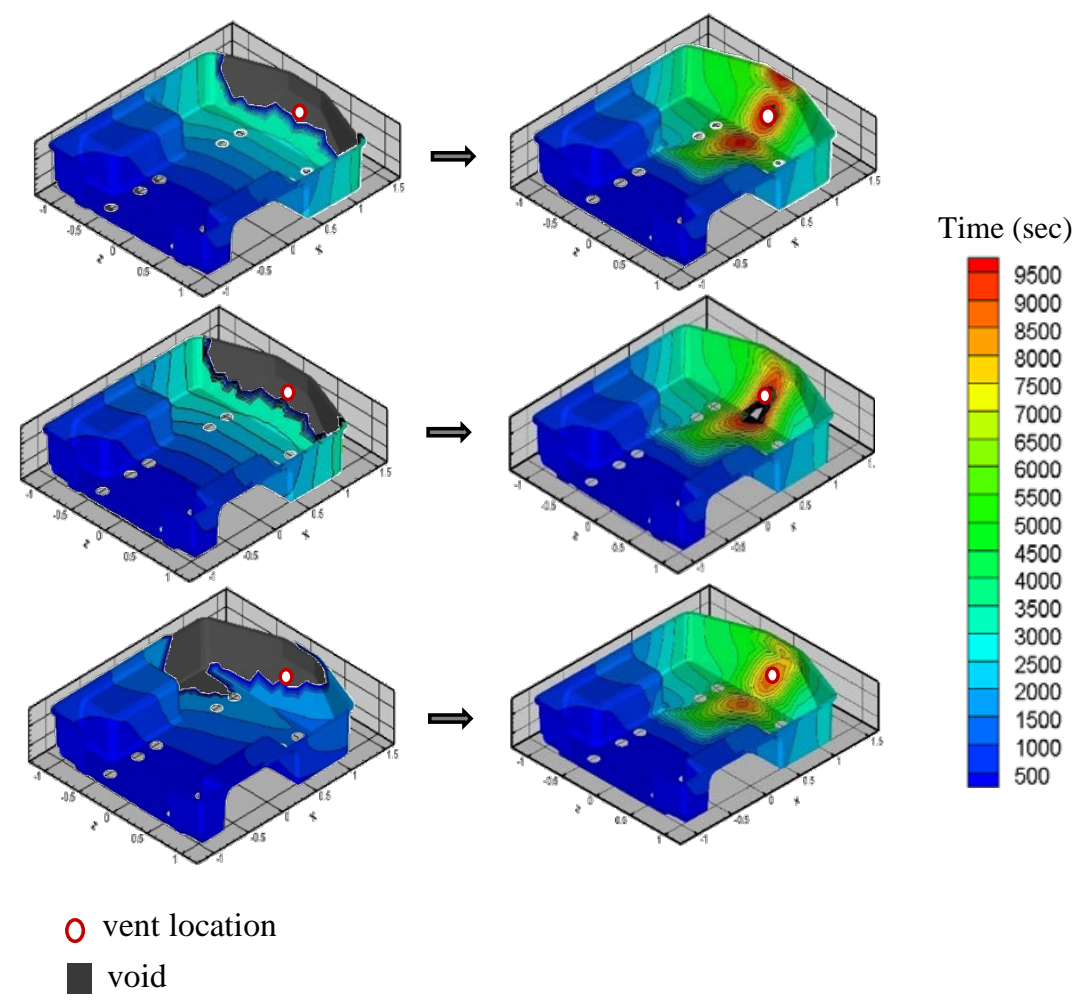

Figure 12. Time contours with full DM on top surface on the left hand side with optimized DM design on the right hand side for three representative scenarios from 1024 possible scenarios 


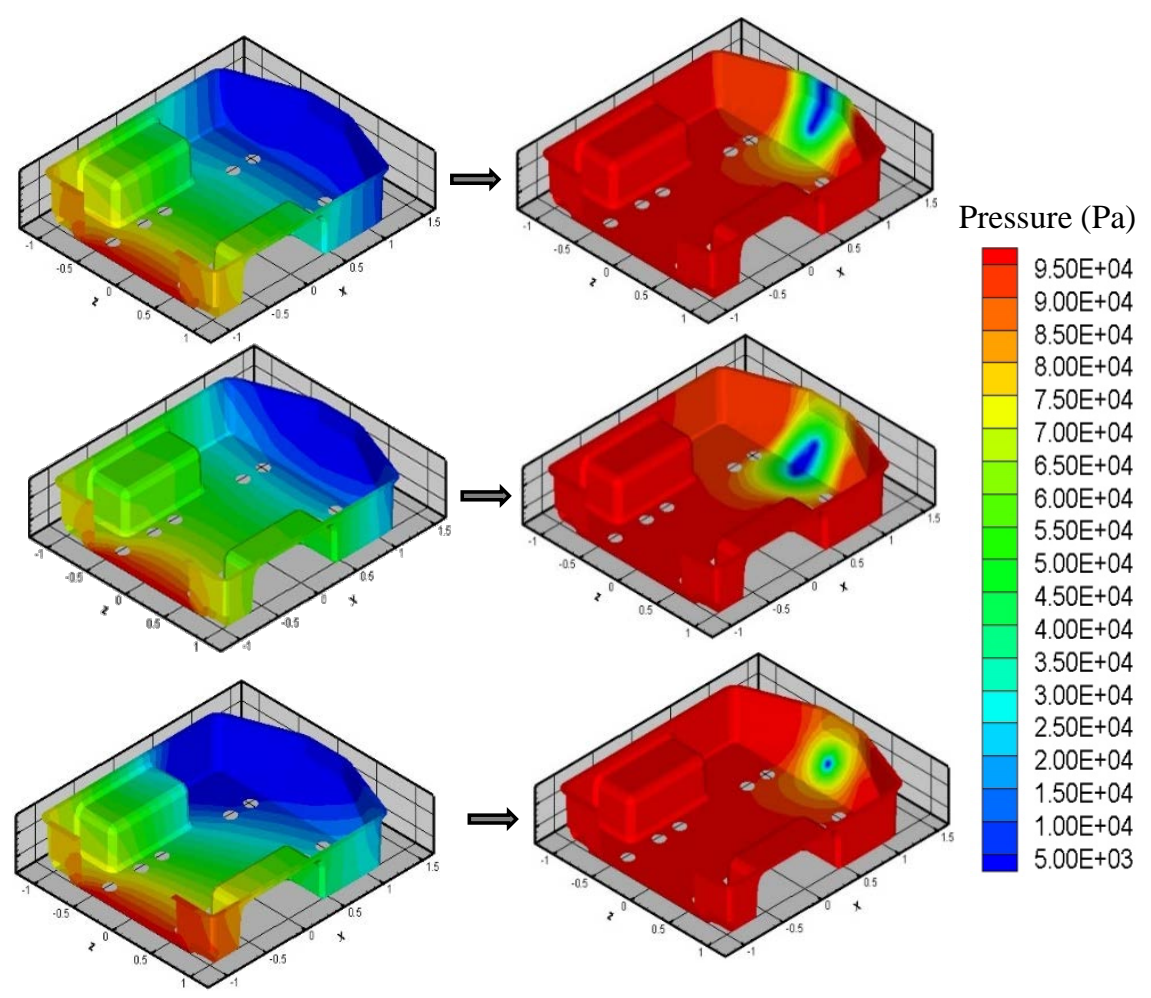

Figure 13. Pressure distribution at the instant resin reaches the vent with full DM on the left and with optimized DM design on the right for the three representative scenarios

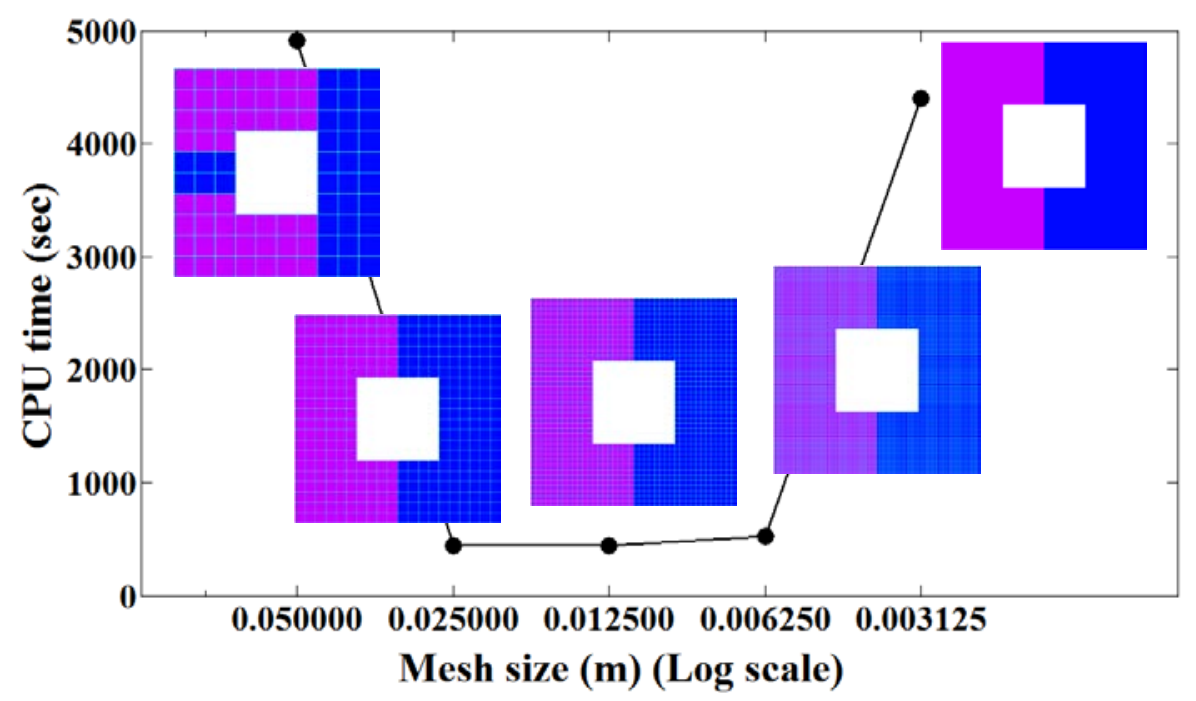

Figure 14. Change in CPU time with mesh size for optimized DM design 


\section{List of Figure Captions}

Figure 1. Tree search algorithms (a) example problem with two acceptable, $\mathrm{H}$ and $\mathrm{T}$, nodes, (b) Breadth-

first search: finds node $\mathrm{H}$, (c) Depth-first search: finds node $\mathrm{T}$

Figure 2. Example to explain the methodology to determine the optimal DM design using the DFS discretization method

Figure 3. Flow chart of the algorithm to obtain optimal DM

Figure 4. Division of the domain with the built in k-means script in Matlab

Figure 5. VARTM test set-up with two cameras system to record the flow front along the top and the bottom surface.

Figure 6. (a) 4th layer of the E-glass with metal insert placed in the center of the fabric, (b) Experiment layup under vacuum with the dark colored region being the DM

Figure 7. DM layout design (a) geometry with inlet/vent locations with 4 race-tracking possibilities along the insert edges creating $2^{4}=16$ different scenarios (b) 8 regions for placement of distribution media when using discrete optimization, and (c) optimum DM design which resulted in successful filling for all 16 scenarios.

Figure 8. Numerical Solution of flow front profiles of the top and bottom views for 4 different racetracking scenarios with time steps 10 seconds apart, (a) with 95\% of the top layer covered with DM, (b) with optimized DM design

Figure 9. Top and bottom flow front profiles: (a) with 95\% DM at the time corn syrup reached the vent (experimental), (b) with optimized DM in 20 seconds apart with the background image of the experiment at 60 seconds (experimental), (c) one of the scenario of numerical simulation in 20 seconds apart

Figure 10. Optimized DM design of trailer geometry with 1024 different possible flow patterns

Figure 11. Void regions with full DM on top surface on the left hand side with optimized DM design on the right hand side for three representative scenarios from 1024 possible scenarios 
Figure 12. Time contours with full DM on top surface on the left hand side with optimized DM design on the right hand side for three representative scenarios from 1024 possible scenarios

Figure 13. Pressure distribution at the instant resin reaches the vent with full DM on the left and with optimized DM design on the right for the three representative scenarios

Figure 14. Change in CPU time with mesh size for optimized DM design 